\title{
Simultaneous all-optical demultiplexing and regeneration based on self-phase and cross-phase modulation in a dispersion shifted fiber
}

\author{
Yu, Jianjun; Jeppesen, Palle
}

Published in:

Journal of Lightwave Technology

Link to article, DOI:

$10.1109 / 50.933288$

Publication date:

2001

Document Version

Publisher's PDF, also known as Version of record

Link back to DTU Orbit

Citation (APA):

Yu, J., \& Jeppesen, P. (2001). Simultaneous all-optical demultiplexing and regeneration based on self-phase and cross-phase modulation in a dispersion shifted fiber. Journal of Lightwave Technology, 19(7), 941-949. https://doi.org/10.1109/50.933288

\section{General rights}

Copyright and moral rights for the publications made accessible in the public portal are retained by the authors and/or other copyright owners and it is a condition of accessing publications that users recognise and abide by the legal requirements associated with these rights.

- Users may download and print one copy of any publication from the public portal for the purpose of private study or research.

- You may not further distribute the material or use it for any profit-making activity or commercial gain

- You may freely distribute the URL identifying the publication in the public portal 


\title{
Simultaneous All-Optical Demultiplexing and Regeneration Based on Self-Phase and Cross-Phase Modulation in a Dispersion Shifted Fiber
}

\author{
Jianjun Yu and Palle Jeppesen, Member, IEEE
}

\begin{abstract}
Simultaneous demultiplexing and regeneration of 40-Gb/s optical time division multiplexed (OTDM) signal based on self-phase and cross-phase modulation in a dispersion shifted fiber is numerically and experimentally investigated. The optimal walkoff time between the control pulse and OTDM signal is obtained by numerical simulation. Our experiment also shows that it is an effective method for realizing simultaneous demultiplexing and regeneration when used in the middle of a system or in the receiver with a proper walkoff time.
\end{abstract}

Index Terms-All-optical demultiplexing, all-optical regeneration, all-optical wavelength conversion, cross-phase modulation (XPM), self-phase modulation (SPM).

\section{INTRODUCTION}

H IGH-capacity all-optical networks based on optical time division multiplexing (OTDM) and wavelength division multiplexing (WDM) require compact and stable switching devices, for example for high-speed demultiplexing and add-drop multiplexing [1]-[5]. Many high-speed demultiplexing methods and devices have been demonstrated, such as cross-phase modulation (XPM), in a nonlinear optical loop mirror (NOLM) [2], semiconductor optical amplifier (SOA), Mach-Zehnder interferometer (MZI), or Michelson interferometer [3], [4], four-wave mixing in a dispersion shifted fiber (DSF), or an SOA [5] and electroabsorption (EA) modulator. Using these demultiplexing methods, the degradation of signal quality due to noise accumulation, pulse distortion, and crosstalk cannot be effectively suppressed. All-optical $2 \mathrm{R}$ regeneration based on the effect of self-phase modulation (SPM) of the data signal in a dispersion shifted fiber has been demonstrated [6]. This method suppresses the noise in 0 bits and the amplitude fluctuations in 1-bits of return-to-zero (RZ) optical data streams. Use of XPM with subsequent filtering to realize wavelength conversion was reported in [7]; recently $40-\mathrm{Gb} / \mathrm{s}$ wavelength conversion was realized by using this method [8]. In this paper, based on the two methods of [6] and [7], we present a new method that has the function of simultaneous demultiplexing and regeneration. The method is

Manuscript received March 6, 2000; revised November 8, 2000

J. Yu was with the Research Center COM, Technical University of Denmark, Lyngby DK-2800, Denmark. He is now with Agere Systems, Murray Hill, NJ 07974 USA (e-mail: jianjun@agere.com).

P. Jeppesen is with the Research Center COM, Technical University of Denmark, Lyngby DK-2800, Denmark (e-mail: pj@com.dtu.dk).

Publisher Item Identifier S 0733-8724(01)05314-2. based on the effects of XPM and SPM in a dispersion shifted fiber after subsequent optical filtering demultiplexing and regeneration are realized simultaneously.

This paper is organized as follows. Section II presents the principle for simultaneous all-optical demultiplexing and regeneration. In Section III, numerical simulations with different walkoff times between control pulses and OTDM signals are presented and the optimal walkoff time is obtained. In Section IV ,we describe two experiments. The first one shows that the method can be used in the middle of a transmission system. The regenerated and dropped channel signal has small chirp and almost the same pulsewidth as the input signal and hence is suitable for further transmission when re-added in other OTDM streams. The second experiment verifies that the method has a strong capacity for noise suppression when the dropped channel is directly used in the receiver. Our experiments also show that simultaneous all-optical demultiplexing and regeneration can be realized only for proper walkoff time. Finally, Section V summarizes the results.

\section{PRINCIPLE}

Qualitatively, the idea of the method is as follows. When the control pulses and the OTDM signals propagate simultaneously in a DSF, the optical spectrum of the OTDM signals will be broadened because of SPM and XPM. Assuming that the input OTDM signals have the spectral bandwidth $\Delta \omega_{0}$ and that dispersion can be ignored, the optical spectrum of the desired channel to be demultiplexed from the OTDM signals broadens due to SPM and XPM

$$
\omega_{\mathrm{SPM} / \mathrm{XPM}}=\Delta \omega_{0}(2 \pi / \lambda) n_{2} I_{s} L+2 \Delta \omega_{0}(2 \pi / \lambda) n_{2} I_{c} L
$$

Here $I_{s}$ and $I_{c}$ are the pulse intensity of signal and control pulses, respectively, $n_{2}$ the nonlinear refractive index, $\lambda$ the wavelength of the OTDM signals, and $L$ the length of the DSF.

The other channels in the aggregated OTDM signal only suffer SPM; therefore, the optical spectrum of the other channels after propagation in the DSF will broaden according to

$$
\omega_{\mathrm{SPM}}=\Delta \omega_{0}(2 \pi / \lambda) n_{2} I_{s} L
$$

Equation (1) is the same as (2) except that an extra frequency shift caused by XPM is added in (1). Because of the XPM, the optical spectrum of the desired channel to be demultiplexed will be separated in frequency from that of other channels. After the 


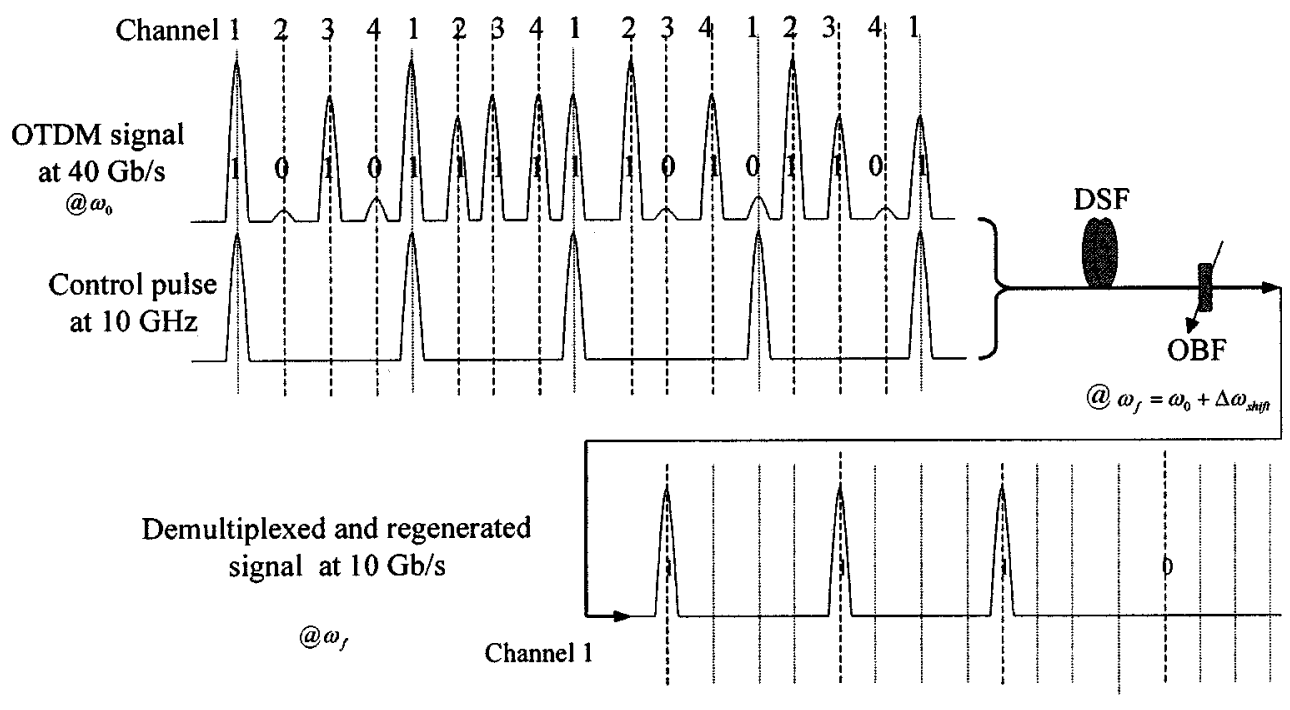

Fig. 1. Schematic diagram of simultaneous demultiplexing and regeneration.

DSF, the signals pass through an optical filter, whose center frequency, $\omega_{f}$, is shifted $\Delta \omega_{\text {shift }}$ with respect to the input OTDM signal frequency $\omega_{0}$

$$
\omega_{f}=\omega_{0}+\Delta \omega_{\text {shift }}
$$

If the spectral broadening (1) of a pulse is small enough, for example when

$$
\omega_{\mathrm{SPM} / \mathrm{XPM}} / 2<\Delta \omega_{\text {shift }}
$$

then the pulse is rejected by the filter. This happens when the pulse intensity $I_{s}$ is too small (noise in 0 bits). If the control pulse intensity is high enough (1 bit) so that

$$
\omega_{\mathrm{SPM} / \mathrm{XPM}} / 2 \geq \Delta \omega_{\text {shift }}
$$

then a part of the XPM- and SPM-broadened spectrum passes through the filter. The bandwidth of the filtered pulse is determined by the filter bandwidth $\Delta \omega_{f}$. In this way simultaneous demultiplexing and regeneration is realized.

Fig. 1 shows an example in which a $10-\mathrm{Gb} / \mathrm{s}$ signal is demultiplexed and regenerated simultaneously from an aggregated 40-Gb/s OTDM signal. The 40-Gb/s OTDM signal consists of four channels at $10 \mathrm{~Gb} / \mathrm{s}$ each, channel 1 consists of $1110 \cdots$, channel 2 of $0111 \cdots$, and so on. We discuss the case in which channel 1 is demultiplexed and regenerated. It is clearly seen that the signals in the other channels will not suffer XPM; however, the 1-bits in channel 1 will suffer XPM in addition to SPM, which causes the spectrum of the 1-bits in channel 1 to be strongly broadened. So, the spectrum of the 1-bit in channel 1 is separated from those of other channels. The first and second 1-bit in channel 1 have a large intensity, so they will suffer stronger SPM than the third 1-bit in channel 1. However, if we choose the center frequency of the optical filter properly, we can ensure that only part of the XPM- and SPM-broadened spectrum passes through the filter. In this way the intensity of the first and second 1-bit becomes the same as that of the third 1-bit after demultiplexing. Furthermore, we can see that the first 0-bit in channel 1 is represented by a small pulse, but such a pulse will only suffer small SPM and XPM, so after the optical filter the small pulse is rejected. In this way channel 1 is demultiplexed and regenerated simultaneously.

The control pulse and the signal of the desired channel to be demultiplexed will be separated in time after the signal and control pulse have propagated a certain distance in the DSF if the walkoff time between the signal and control pulse is not zero. The walkoff effect reduces XPM; hence the spectrum of the desired channel to be demultiplexed might not be separated effectively from those of other channels. Therefore, we must choose a DSF with proper zero dispersion wavelength in order to make the walkoff time small. However, in the following numerical simulations and experiments we will see that there is no regeneration capacity if the walkoff time is very small. So, we must choose a proper compromise for the walkoff time in order to realize simultaneous all-optical regeneration and demultiplexing. We will analyze in detail some special cases by numerical simulation in the following section.

\section{NUMERICAL SIMULATIONS}

The propagation of the control pulses and the OTDM signals in the 15-km DSF is governed by the nonlinear Schrödinger equation (NLSE). Group velocity dispersion (GVD), SPM, and XPM are incorporated into our model.

The propagation of the control pulses and OTDM signals in the DSF can be expressed by the following coupled NLSEs [2]: Control pulse:

$$
\begin{aligned}
& \frac{\partial A_{1}}{\partial z}+\frac{1}{\nu_{1}} \frac{\partial A_{1}}{\partial t}+\frac{i}{2} \beta\left(\lambda_{1}\right) \frac{\partial^{2} A_{1}}{\partial t^{2}}+\frac{1}{2} \alpha A_{1} \\
& =i \gamma_{1}\left[\left|A_{1}\right|^{2}+2\left|A_{2}\right|^{2}\right] A_{1}
\end{aligned}
$$

OTDM pulse:

$$
\begin{aligned}
& \frac{\partial A_{2}}{\partial z}+\frac{1}{\nu_{2}} \frac{\partial A_{2}}{\partial t}+\frac{i}{2} \beta\left(\lambda_{2}\right) \frac{\partial^{2} A_{2}}{\partial t^{2}}+\frac{1}{2} \alpha A_{2} \\
& =i \gamma_{2}\left[2\left|A_{1}\right|^{2}+\left|A_{2}\right|^{2}\right] A_{2} .
\end{aligned}
$$




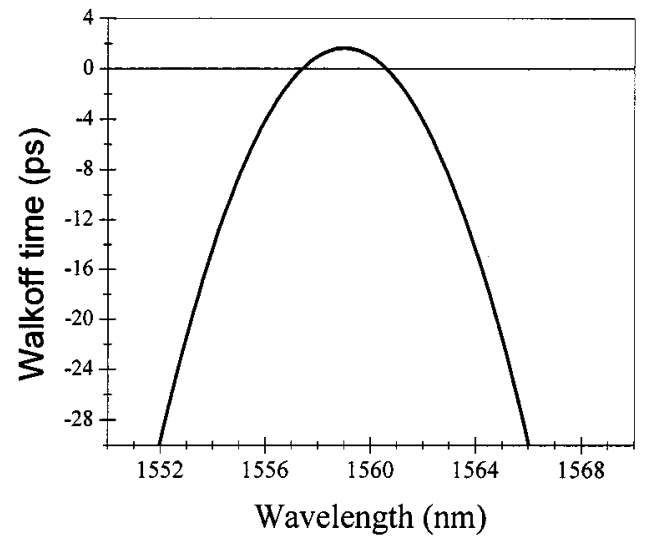

Fig. 2. Calculated walkoff time between the control pulse at $1560.6 \mathrm{~nm}$ and OTDM pulse at different wavelengths.

Here, $\lambda_{i}(i=1,2)$ is the wavelength, $A_{i}$ is the propagating optical field amplitude, $\nu_{i}$ the group velocity, $\beta\left(\lambda_{i}\right)$ the linear group velocity dispersion parameter given by $-\lambda_{i}^{2} D_{i} / 2 \pi c$ where $D_{i}$ is the dispersion parameter and $c$ is the speed of light in vacuum, $\gamma_{i}$ the nonlinear coefficient defined by $\gamma_{i}=n_{2} \omega_{i} / c A_{\text {eff }}=2 \pi n_{2} / A_{\text {eff }} \lambda_{i}$, where the nonlinear index coefficient $n_{2}=2.67 \times 10^{-20} \mathrm{~m}^{2} / \mathrm{W}$, and $A_{\mathrm{eff}}=50 \mu \mathrm{m}^{2}$ is the effective fiber core area. $\alpha=0.25 \mathrm{~dB} / \mathrm{km}$ is the loss of the DSF, and we assume that it is independent of wavelength. The shapes of the control and signal pulses are both Gaussian. The peak power of the control pulse is $400 \mathrm{~mW}$. The FWHM pulsewidths of the control pulse and signal are both 8 ps. The DSF has a length of $15 \mathrm{~km}$ with zero dispersion wavelength of $1559 \mathrm{~nm}$ and dispersion slope of $0.08 \mathrm{ps} / \mathrm{nm}^{2} / \mathrm{km}$. Fig. 2 shows the calculated walkoff time between the control pulse at $1560.6 \mathrm{~nm}$ and OTDM pulse at different wavelengths.

First, we investigate the optical spectrum characteristics for the case where the wavelengths of the control pulse and signals are 1560.6 and $1555.7 \mathrm{~nm}$, respectively.

Fig. 3 shows the optical spectra for different input signal power. From Fig. 3 we can obtain the following conclusions: 1) 3-dB bandwidth of the original OTDM signal spectrum is approximately $100 \mathrm{GHz}(0.8 \mathrm{~nm}) ; 2)$ new spectral components of the OTDM signal are generated because of SPM when the peak power $P_{p}$ of the OTDM signals exceeds about $100 \mathrm{~mW} ; 3$ ) considering the desired channel to be demultiplexed, the optical spectrum of the 1-bits of this channel is broadened because of XPM, and the frequency of the peak of the spectrum is shifted to a higher frequency and the shape of the optical spectrum is asymmetrical; and 4) the larger $P_{p}$, the larger the frequency shift of the peak of the spectrum.

We use an optical filter to filter the optical spectrum after the control pulses and the OTDM signals have propagated over the DSF. The bandwidth (FWHM) of the Gaussian optical filter is $0.8 \mathrm{~nm}$ and the frequency shift of the optical filter $\left(\Delta \omega_{\text {shift }} / 2 \pi\right)$ to a higher frequency than the OTDM signal is $100 \mathrm{GHz}$. It is clearly seen that the output signal is zero for small input signal. Fig. 4 shows the transfer function when the wavelength of the OTDM signal is $1555.7 \mathrm{~nm}$. Fig. 4 demonstrates that it is a very effective method for suppressing the small signal noise, such as ASE noise accumulation. When $P_{p}$ is in the 75 to $150 \mathrm{~mW}$

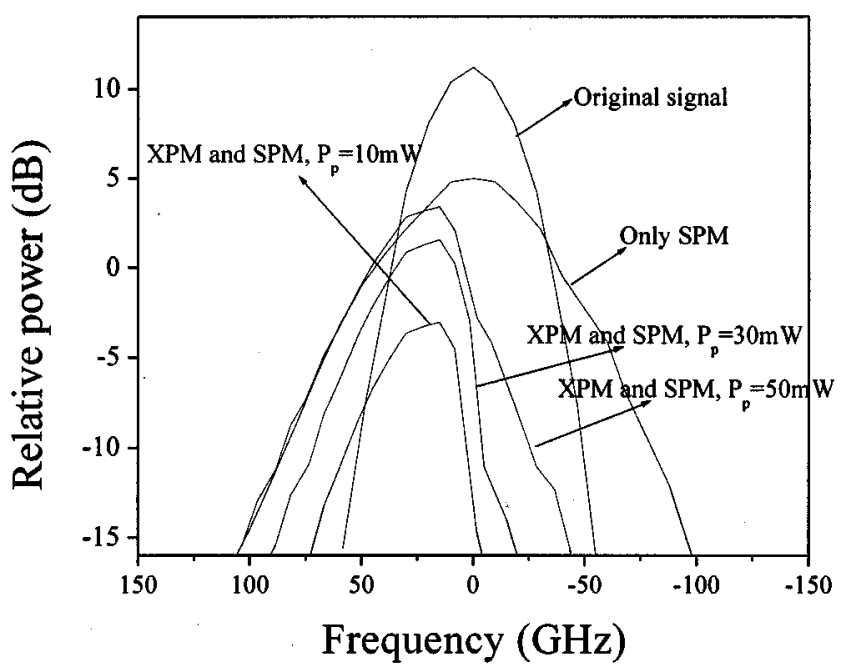

(a)

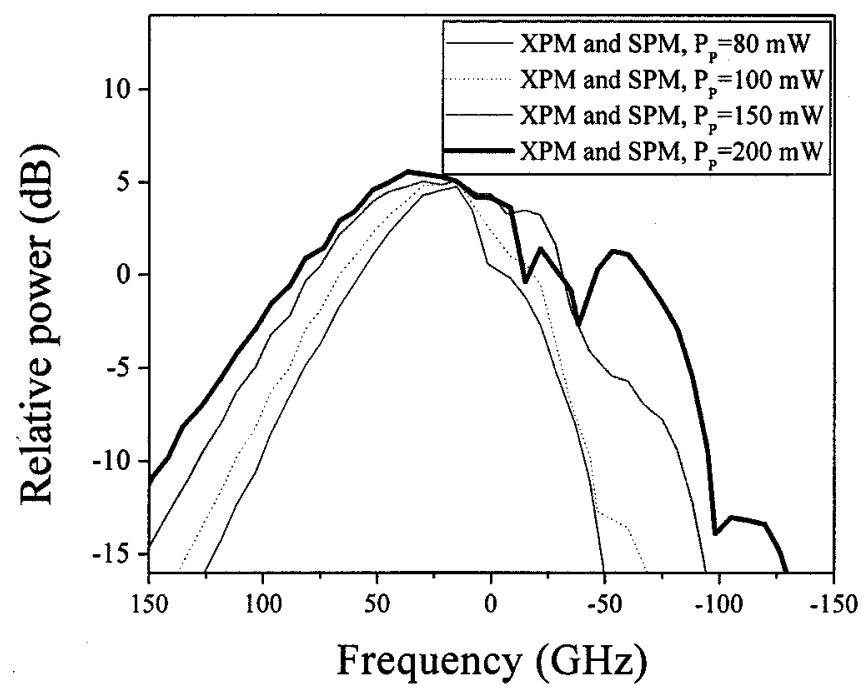

(b)

Fig. 3. Optical spectra of " 1 " in the signal at $1555.7 \mathrm{~nm}$ with the control pulse at $1560.6 \mathrm{~nm}$. (a) Original signal, only considering SPM, considering both SPM and XPM with $P_{p}$ of $10 \mathrm{~mW}, 30 \mathrm{~mW}$, and $50 \mathrm{~mW}$, respectively. (b) Considering both SPM and XPM with $P_{p}$ of $80 \mathrm{~mW}, 100 \mathrm{~mW}, 150 \mathrm{~mW}$, and $200 \mathrm{~mW}$, respectively.

range, the change in the output intensity is small; in this case, the method shows some regeneration capacity for 1-bits. When the signal is large, such as $P_{p}$ larger than $150 \mathrm{~mW}$, the broadened spectrum of the other channels cannot be effectively separated from that of the desired channel. From Fig. 4, we can see that the output power increases very fast when $P_{p}$ becomes larger than $150 \mathrm{~mW}$; this demonstrates that there is only a weak regeneration capacity when the power in the 1-bits becomes too large.

In the following section we will consider the case where the wavelengths of the control pulses and the OTDM signals are 1560.6 and $1557.3 \mathrm{~nm}$, respectively.

When the wavelength of the OTDM signals is $1557.3 \mathrm{~nm}$, the optical spectrum for $P_{p}$ of $100 \mathrm{~mW}$ and only considering SPM is shown in Fig. 5. We can see that the optical spectrum is almost the same as in Fig. 3(a) because in both cases GVD is small and SPM is the main effect. However, when XPM is considered, the optical spectrum of the signals is divided into 


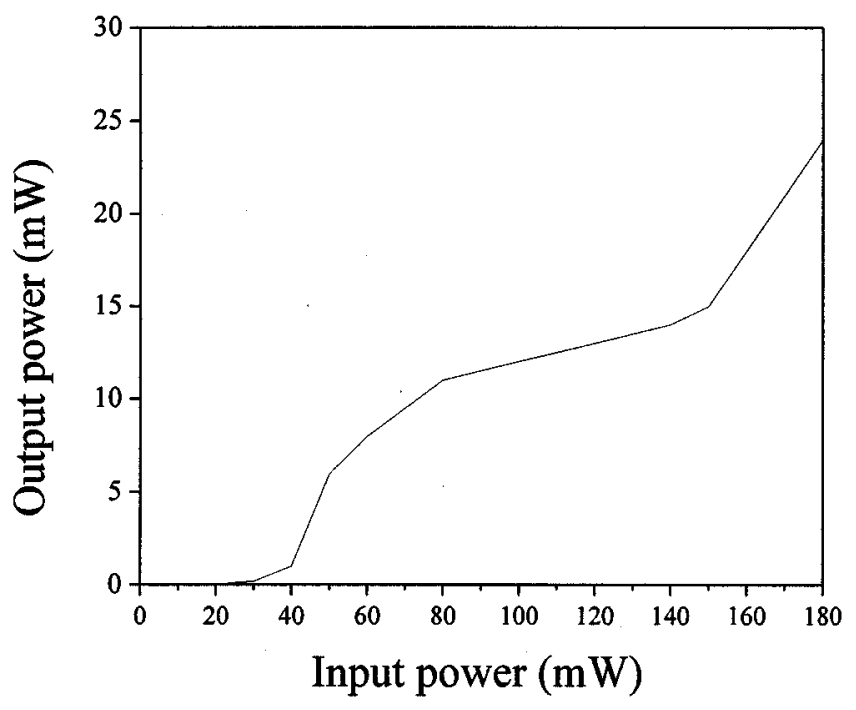

Fig. 4. Transfer function for the wavelength of the OTDM signals at $1555.7 \mathrm{~nm}$.

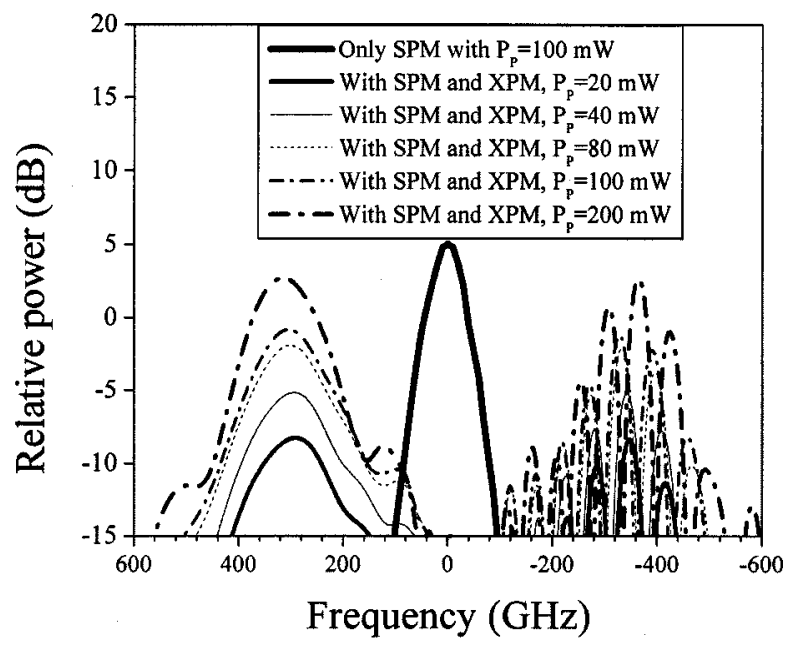

Fig. 5. Optical spectrum of " 1 " in the signal at $1557.3 \mathrm{~nm}$ with the control pulse at $1560.6 \mathrm{~nm}$. (a) Only considering SPM, simultaneously considering XPM and SPM with $P_{p}=20 \mathrm{~mW}, 40 \mathrm{~mW}, 80 \mathrm{~mW}, 100 \mathrm{~mW}$, and $200 \mathrm{~mW}$, respectively.

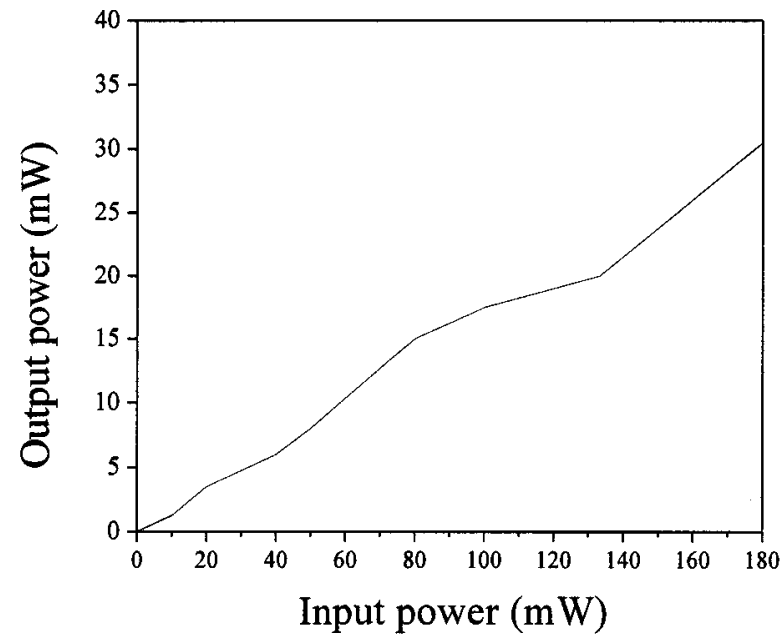

Fig. 6. Transfer function for the wavelength of OTDM signals at $1557.3 \mathrm{~nm}$.

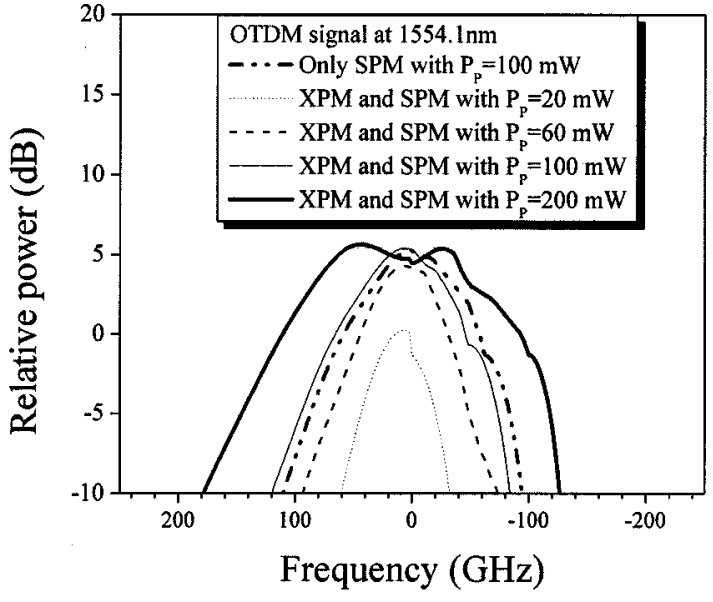

(a)

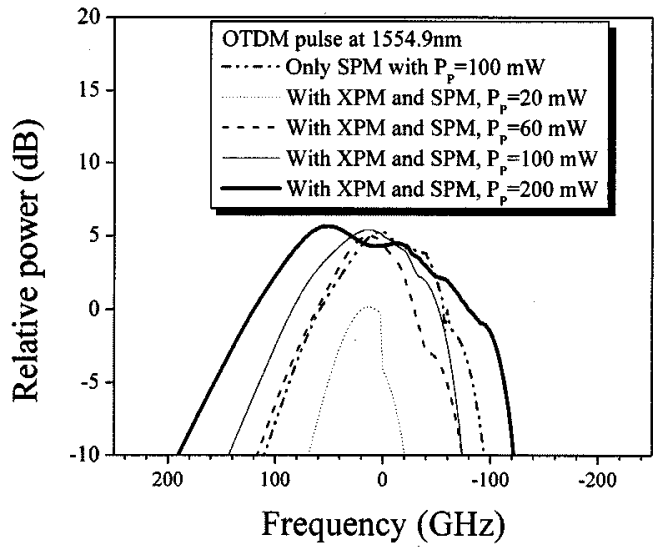

(b)

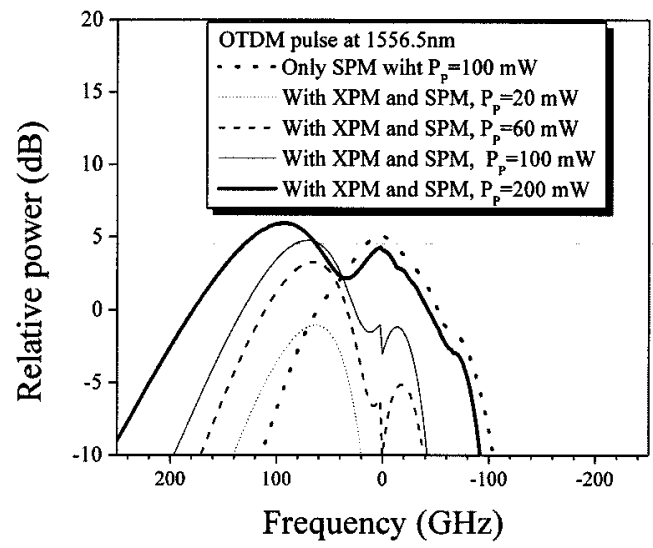

(c)

Fig. 7. Optical spectrum of "1" in the signal with the control pulse at 1560.6 $\mathrm{nm}$ and for different wavelength OTDM pulse: (a) $1554.1 \mathrm{~nm}$, (b) 1554.9 $\mathrm{nm}$, and (c) $1556.5 \mathrm{~nm}$.

two sections because a small walkoff leads to a strong XPM; the peak wavelength is further shifted to the higher frequency side and in the right part of the optical spectrum appears a multipeak configuration [10]. The optical spectra for $P_{P}$ chosen in the 20 to $200 \mathrm{~mW}$ range are also shown in Fig. 5. We can see that the frequency shift of the peak of the spectra is $270 \mathrm{GHz}$ and almost independent of $P_{p}$. When $\Delta \omega_{\text {shift }} / 2 \pi$ is $270 \mathrm{GHz}$ and the shift is toward higher frequency, it is easy to filter the optical spectrum of the desired channel to be demultiplexed. Fig. 6 shows the 


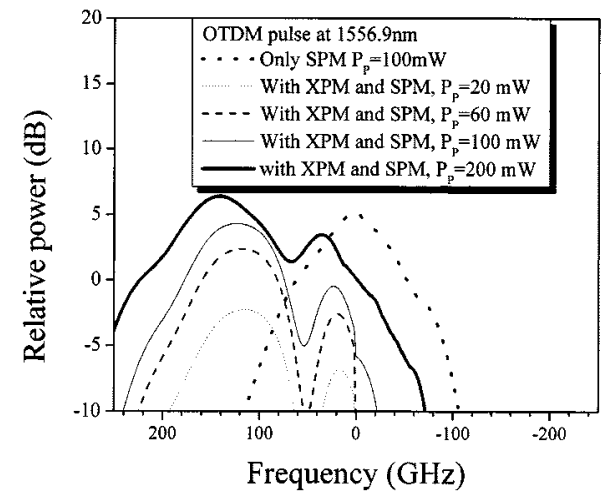

(d)

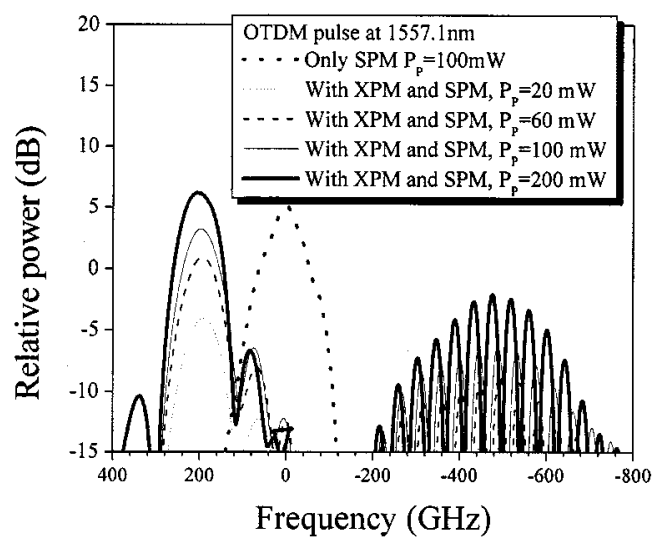

(e)

Fig. 7 (Continued). Optical spectrum of "1" in the signal with the control pulse at $1560.6 \mathrm{~nm}$ and for different wavelength OTDM pulse: (d) $1556.9 \mathrm{~nm}$ and (e) $1557.1 \mathrm{~nm}$.

transfer function when the wavelength of the OTDM signals is $1557.3 \mathrm{~nm}$. The parameters are: the bandwidth (FWHM) of the Gaussian optical filter is $0.8 \mathrm{~nm}$ and $\Delta \omega_{\text {shift }} / 2 \pi$ is $270 \mathrm{GHz}$. Fig. 6 shows an almost linear relationship between the input and output power demonstrating that there is no regeneration capacity in this case.

Comparing the above two cases, we can see that there is a regeneration capacity only when the walkoff time is greater than a certain value. In the following section, we numerically investigate the fact that the regenerative capacity of the demultiplexing is lost at certain values of the walkoff time. Fig. 7 shows the optical spectra for different wavelengths OTDM signals. When the wavelength of the OTDM signals is $1554.1 \mathrm{~nm}$, we can see from Fig. 2 that the walkoff time is 15 ps. Because the walkoff time is too great, there is only a small broadened spectrum, which leads to the fact that the spectrum of the desired channel cannot be effectively separated from that of the other channels. In such a case, we cannot realize all-optical demultiplexing and regeneration. When the wavelength of the OTDM signal is $1554.9 \mathrm{~nm}$, the walkoff time is $9.4 \mathrm{ps}$. In this case, the demultiplexing can be realized, but the regeneration capacity is not good. When the wavelength of OTDM signals are 1556.5 and $1556.9 \mathrm{~nm}$, the walkoff times are 3 and 2 ps, respectively. In these two cases, the optical spectra are similar to that in Fig. 3, and the demultiplexing and regeneration can be effectively realized. However, when the wavelength of the OTDM signals is $1557.1 \mathrm{~nm}$ and the walkoff time is $1 \mathrm{ps}$, the optical spectrum is similar to that in Fig. 5. In this case, demultiplexing is easily realized, but not regeneration. It means that in order to realize simultaneous demultiplexing and regeneration, the walkoff time should be smaller than 9.4 ps and larger than 1 ps.

\section{EXPERIMENTAL RESULTS AND DISCUSSION}

It is well known that the dropped channel from an OTDM stream can be used either to add to another OTDM steam and then be transmitted a long distance [9] or to be received by a receiver in an optical network. If it has to be re-added into another OTDM stream, not only the regeneration capacity but also the chirp and pulsewidth characteristics are very important. However, if the demultiplexed channel is just received by a receiver, the regeneration capacity is very important.

\section{A. Application in the Middle of a System}

The experimental setup is shown in Fig. 8. The 10-Gb/s RZ signals at 1555.7 or $1557.3 \mathrm{~nm}$ are obtained by the use of wavelength conversion based on a NOLM [11], [12]. The control laser for wavelength conversion is a $10 \mathrm{GHz}, 1560.6-\mathrm{nm}$ gainswitched distributed feedback laser diode that generates 8 ps (FWHM) pulses after compression in a dispersion compensation fiber (DCF). After adding the $\mathrm{LiNbO}_{3}$ modulator, and modulating the control pulses with a pseudorandom bit sequence of $2^{31}-1$, a control signal at $10 \mathrm{~Gb} / \mathrm{s}$ is obtained. The continuous wave $(\mathrm{CW})$ lightwave is generated by a DFB-LD at 1555.7 or $1557.3 \mathrm{~nm}$. The control signal is coupled into the NOLM using a wavelength independent $3-\mathrm{dB}$ optical coupler. A polarization controller in the loop is used to obtain best performance of the converted pulses [11]. A 1.6-nm bandpass tunable optical filter at the output of the NOLM is used to suppress the control pulses. All optical couplers are $2 \times 2$ couplers with power coupling ratios of 50: 50\%. The NOLM for wavelength conversion consists of $3 \mathrm{~km}$ of DSF with zero dispersion wavelength of $1550.7 \mathrm{~nm}$ and dispersion slope of $0.08 \mathrm{ps} / \mathrm{nm}^{2} / \mathrm{km}$. The converted signals at 1555.7 or $1557.3 \mathrm{~nm}$ are multiplexed to $40 \mathrm{~Gb} / \mathrm{s}$ by a fiber delay-line multiplexer. The aggregated 40-Gb/s OTDM signal is then amplified to $10-\mathrm{dBm}$ average power by an EDFA, it is compensated by $4.8-\mathrm{km}$ DCF after transmission through $30-\mathrm{km}$ single mode fiber (SMF), then amplified to $10 \mathrm{dBm}$ again, transmitted through another 25-km SMF and 3.6-km DCF, then amplified to $18 \mathrm{dBm}$ again and finally transmitted through another 50-km SMF and 7.2-km DCF. The DCFs have a negative dispersion slope, the higher order dispersion is also compensated besides group velocity dispersion, and the fiber chain has almost full-dispersion compensation. The demultiplexing in the time domain is realized by use of a DSF with a length of $16 \mathrm{~km}$, zero dispersion wavelength of $1559 \mathrm{~nm}$, and dispersion slope of $0.08 \mathrm{ps} /\left(\mathrm{nm}^{2} \cdot \mathrm{km}\right)$. The powers of the control pulses and the 40-Gb/s OTDM signal are each adjusted by a tunable attenuator. However, the total power into the DSF is maintained to $18 \mathrm{dBm}$. Adjusting the optical time-delay of the control pulses allows us to demultiplex the desired channel from the $40-\mathrm{Gb} / \mathrm{s}$ OTDM stream. The optical spectrum of the demultiplexed channel is filtered by two cascaded tunable optical filters. 


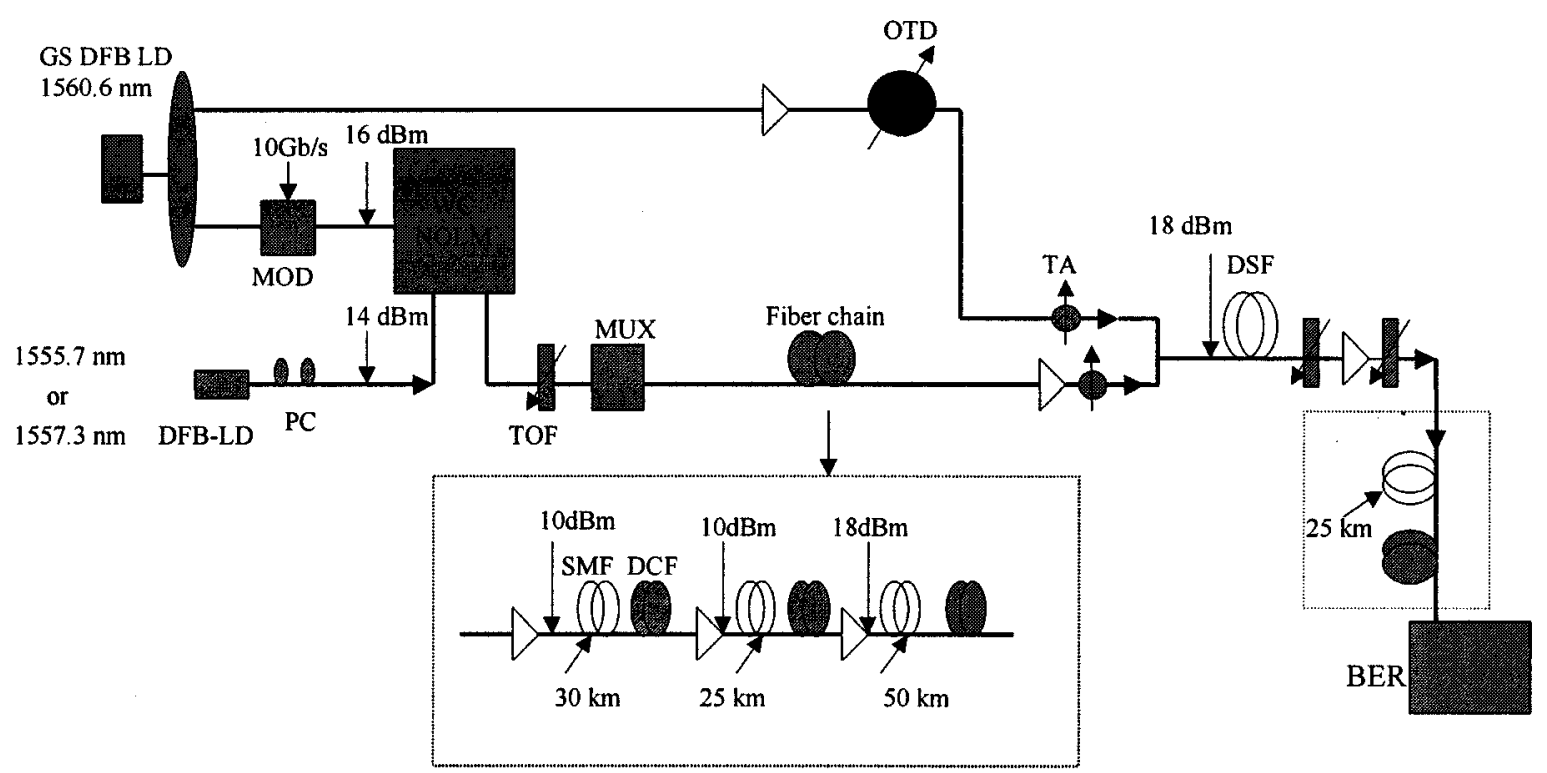

Fig. 8. Experimental setup for studying application in the middle of a system.

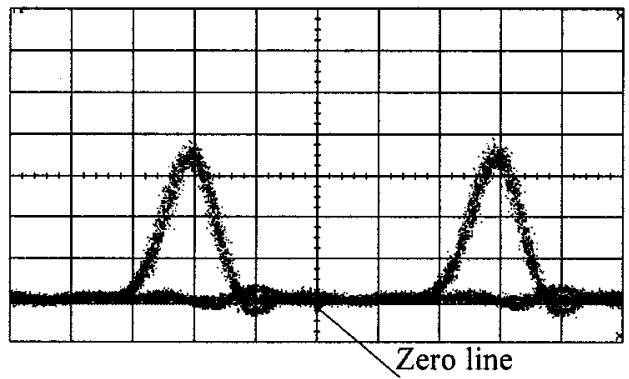

(a)

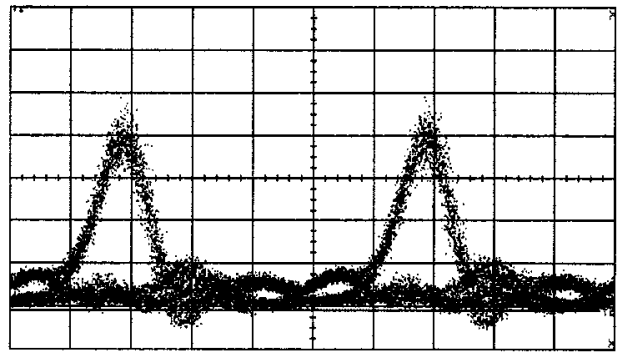

(c)

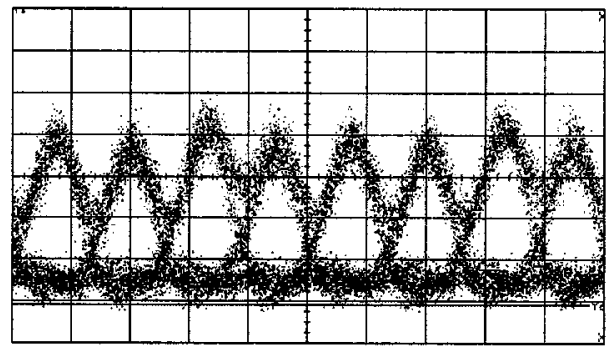

(b)

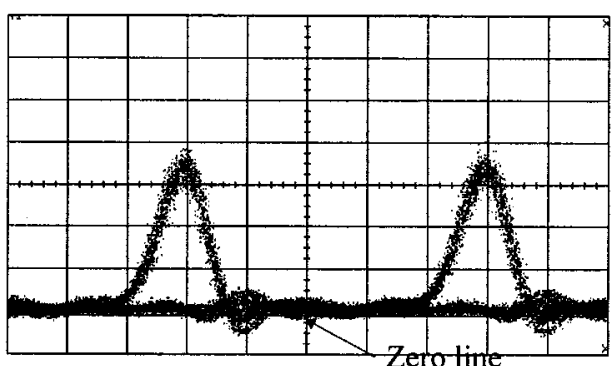

(d)

Fig. 9. Eye diagrams at $1555.7 \mathrm{~nm}$. (a) Converted signal at $10 \mathrm{~Gb} / \mathrm{s}$. (b) Multiplexed signal before transmission at $40 \mathrm{~Gb} / \mathrm{s}$. (c) Demultiplexed signal at $10 \mathrm{~Gb} / \mathrm{s}$ after transmission over 105-km SMF and the first optical filter. (d) Demultiplexed signal at $10 \mathrm{~Gb} / \mathrm{s}$ after transmission over 105-km SMF and the second optical filter.

First, we let the OTDM signals operate at $1555.7 \mathrm{~nm}$. The eye diagram and BER curve of the 10-Gb/s converted signal are shown in Fig. 9(a) and Fig. 11, respectively. Compared with the BER curve of the original signal at $10 \mathrm{~Gb} / \mathrm{s}$ (back-to-back), the penalty of the converted signal is $0.2 \mathrm{~dB}$ at a BER of $10^{-9}$. The eye diagram of the $40-\mathrm{Gb} / \mathrm{s}$ multiplexed signal is shown in Fig. 9(b). After the OTDM signal is propagated through the DSF, we use a tunable optical filter with a bandwidth of $1.6 \mathrm{~nm}$ to filter the optical spectrum of the OTDM signals. A compar- ison between the optical spectra of the OTDM signals before and after the DSF is shown in Fig. 10. We can see that the optical spectrum of the OTDM signal is broadened because of SPM and XPM. The eye diagram and optical spectrum of the demultiplexed channel after the first optical filter is shown in Fig. 9(c) and Fig. 10, respectively. Because of the limited suppression of the first tunable optical filter, the signals in the other channels can also be seen in Fig. 9(c). We then use the second optical filter to further suppress the signals in the other channels. From 


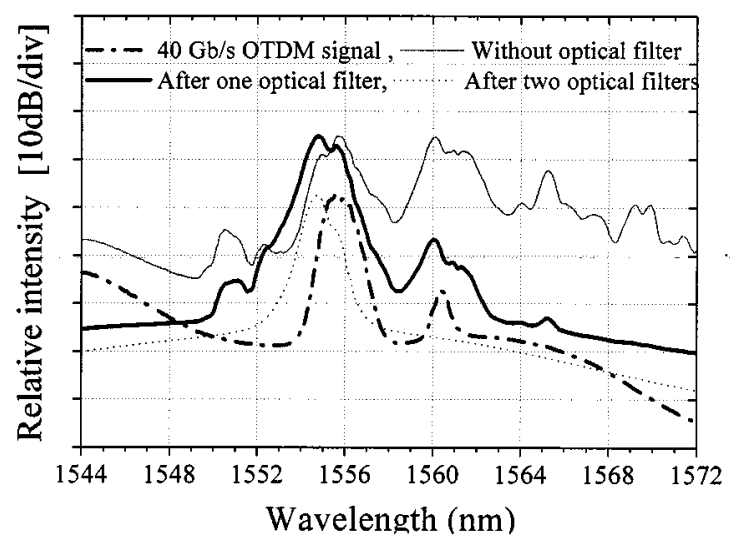

Fig. 10. Optical spectra at $1555.7 \mathrm{~nm}$ after transmission over 105-km SMF (resolution of $0.1 \mathrm{~nm}$ ).

Fig. 9(d) and Fig. 10 we can see that the signals in other channels are effectively suppressed. But, even after two optical filters, some signals in other channels still exist, which lead to a slightly wider zero line in the time slots of the other channels. From Fig. 9(d), we can see that there are almost no fluctuations of 1-bits and 0-bits in the time slots of the desired demultiplexed channel. There is almost no difference between Fig. 9(a) and (d) except for a slightly wider zero line in the time slots of the other channels. The accumulation of ASE noise is suppressed effectively, which demonstrates the regenerative capacity of this method. More BER curves are shown in Fig. 10. Comparing with the BER curve of the converted signal, the penalty of the demultiplexed signal before transmission is $1.1 \mathrm{~dB}$, which is caused by the limited suppression of the optical filter. If we could obtain a narrow bandwidth filter, the penalty could be reduced. We can also let the demultiplexed channel propagate in another DSF like in [5]; by use of SPM occurring when the signals propagate in the DSF, the signals are regenerated again and the other channels are completely suppressed. But after transmission over $105-\mathrm{km} \mathrm{SMF}$, there is no additional power penalty; this shows a very good demultiplexing performance and the ASE noise accumulation is effectively suppressed.

Now we let the OTDM signals operate at $1557.3 \mathrm{~nm}$. Because the zero dispersion wavelength of the DSF is $1559 \mathrm{~nm}$ and the wavelength of the control pulses is $1560.6 \mathrm{~nm}$, there is a minimum walkoff. In that case, the XPM effect is largest, which causes the broadened optical spectrum to be widest. Therefore, in this case, the optical spectrum of the desired channel to be demultiplexed is easy to separate.

The BER curve of the converted signal at $1557.3 \mathrm{~nm}$ is shown in Fig. 11. Compared with the BER curve of the original signal at $10 \mathrm{~Gb} / \mathrm{s}$ (back-to-back), the penalty of the converted signal is $0.2 \mathrm{~dB}$ at a BER of $10^{-9}$. The eye diagram of the $40-\mathrm{Gb} / \mathrm{s}$ OTDM signals at $1557.3 \mathrm{~nm}$ before transmission is shown in Fig. 12(a). Comparing with the BER curve of the converted signals at $10 \mathrm{~Gb} / \mathrm{s}$, the penalty of the demultiplexed signals before transmission is $1 \mathrm{~dB}$. After $105 \mathrm{~km}$ SMF transmission, we demultiplex the $40 \mathrm{~Gb} / \mathrm{s}$ OTDM signals by our new method. Fig. 13 shows the optical spectra after the 40-Gb/s OTDM signals and control pulses have propagated in 16-km DSF. From Fig. 13 we can see that the spectrum of the signals is broadened

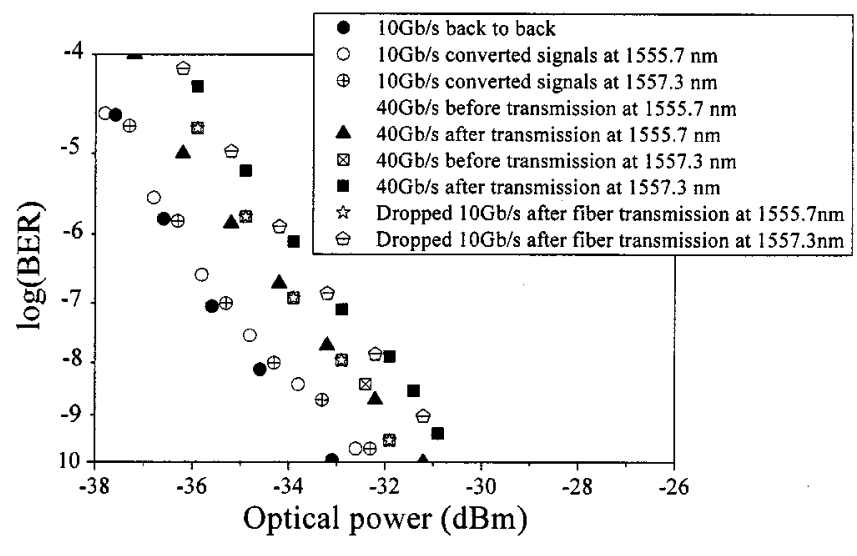

Fig. 11. BER as a function of optical power.

and even split. The shifted frequency of the peak power of the demultiplexed channel is $1.6 \mathrm{~nm}$ and is larger than $0.8 \mathrm{~nm}$ as shown in Fig. 10. After the first tunable optical filter, the eye diagram is shown in Fig. 12(b) and the other channels are almost suppressed. We use the second optical filter to further suppress the other channels and the signals in the other channels are fully suppressed. But we can see that the noise in both the 1-bits and 0-bits in the desired channel is larger than in Fig. 9(d). The BER of the demultiplexed signals after transmission over 105-km SMF is measured and shown in Fig. 11; comparing with the BER curve before transmission, the penalty after transmission is $0.5 \mathrm{~dB}$, which demonstrates that the accumulated ASE noise and pulse distortion by nonlinear effect cannot be suppressed effectively and hence there is no regeneration capacity.

If the dropped signal has to be re-added to other OTDM streams, the chirp characteristic is very important because a chirped signal can cause degradation in a SMF. We let the dropped signal at 1555.7 or $1557.3 \mathrm{~nm}$ be transmitted over 25-km SMF and a DCF providing full dispersion compensation. Then the BER performance is measured and the results are shown in Fig. 11. There are no noticeable added penalties, which demonstrates that the chirp after the demultiplexing is very small. We also measure the pulsewidth after demultiplexing and regeneration and the results are shown in Fig. 14. We can see that the pulsewidth is almost maintained at 8 ps. This demonstrates that the dropped signal is suitable for further transmission in OTDM networks.

\section{B. Application in a Receiver}

When the demultiplexed signal is used in a receiver we will only pay attention to the capacity of noise suppression. Like [13], we change the input signal-to-ASE noise ratio and measure the penalty after demultiplexing and regeneration. The experimental setup is shown in Fig. 15 and it is almost the same as in Fig. 8. No transmission fiber is used and an attenuator before the EDFA and MUX controls the input power and hence the signal-to-ASE noise ratio at the input of the DSF.

The regeneration capacity is demonstrated in Fig. 16. Because of the limited suppression of the filter, there is 1.1-dB penalty when the wavelength of OTDM signals is 1555.7 or $1557.3 \mathrm{~nm}$. We can see that simultaneous demultiplexing and regeneration are realized when the OTDM signal is $1555.7 \mathrm{~nm}$; 


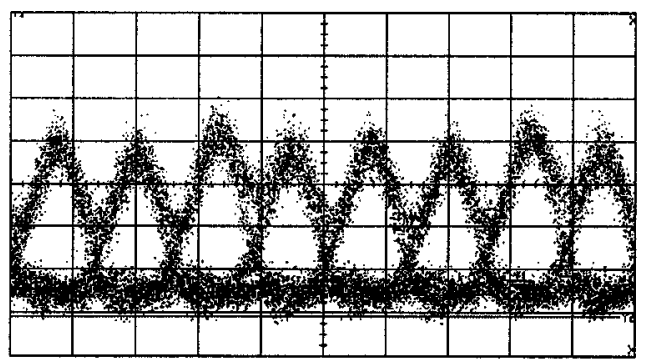

(a)

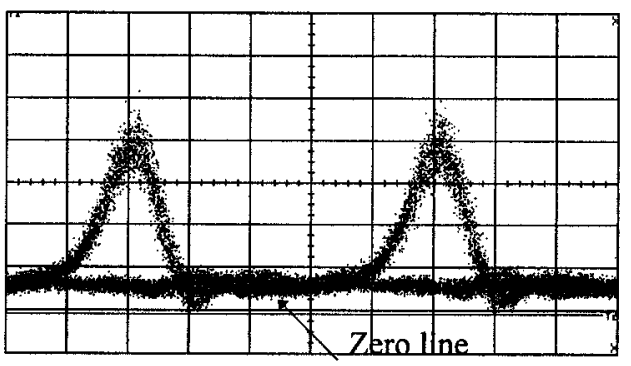

(b)

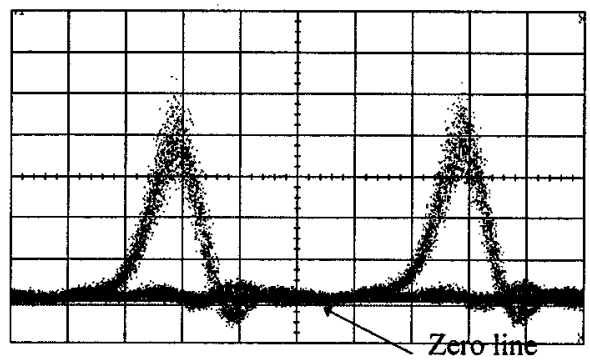

(c)

Fig. 12. Eye diagrams at $1557.3 \mathrm{~nm}$ : (a) Converted signal at $40 \mathrm{~Gb} / \mathrm{s}$, (b) demultiplexed signal at $10 \mathrm{~Gb} / \mathrm{s}$ after transmission over $105-\mathrm{km} \mathrm{SMF}$ and the first optical filter, and (c) demultiplexed signal at $10 \mathrm{~Gb} / \mathrm{s}$ after transmission over 105-km SMF and the second optical filter.

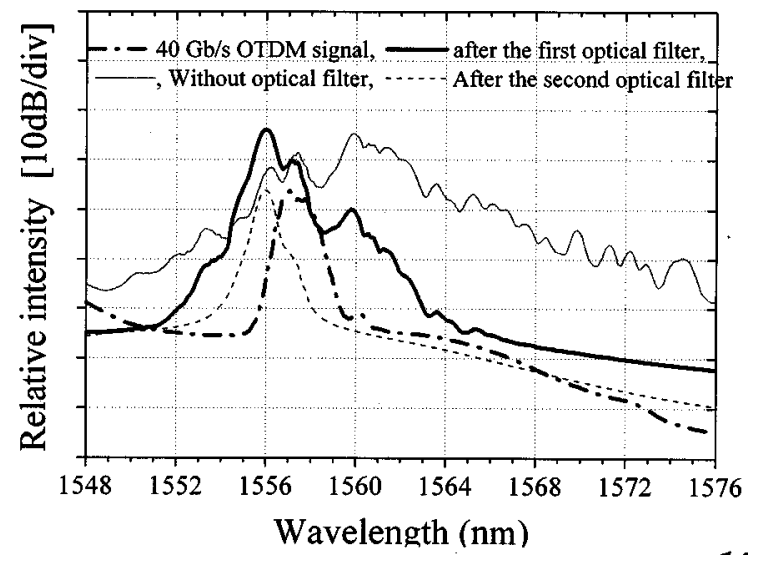

Fig. 13. Optical spectra at $1557.3 \mathrm{~nm}$ after transmission over $105-\mathrm{km}$ SMF (resolution of $0.1 \mathrm{~nm})$.

even when the EDFA input power is as low as $-30 \mathrm{dBm}$, the added penalty is only $1 \mathrm{~dB}$. However, the $10-\mathrm{Gb} / \mathrm{s}$ back-to-back signal at $1555.7 \mathrm{~nm}$ has $3.5-\mathrm{dB}$ penalty for the same EDFA input power. When the OTDM signal wavelength is $1557.3 \mathrm{~nm}$, the ASE noise cannot be effectively suppressed which shows that there is no regeneration capacity.

\section{CONCLUSION}

We have demonstrated a new method for simultaneous OTDM demultiplexing and regeneration. Numerical simulation shows that simultaneous regeneration and demultiplexing of OTDM signals can be realized only when the walkoff time between the control pulses and OTDM signals is larger than $1 \mathrm{ps}$ and smaller than $9.4 \mathrm{ps}$. Our experiments verify the results

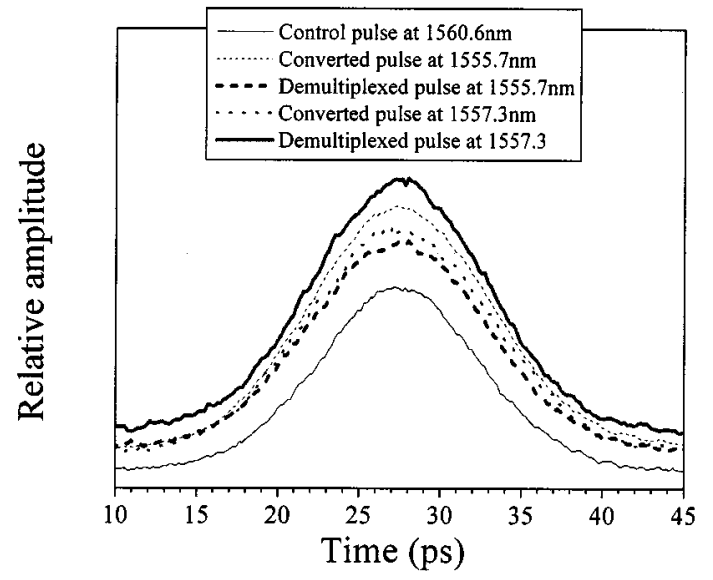

Fig. 14. Measured autocorrelator traces.

from the numerical simulations. The experimental results show that the regenerated and demultiplexed signal has small chirp and its pulsewidth can almost be maintained; hence it is suitable for not only application in a receiver but also for application in the middle of a system where the dropped channel is re-added in other OTDM streams.

\section{ACKNOWLEDGMENT}

The authors would like to thank Lucent Technologies Denmark for providing the SMFs, DSFs, and DCFs, and France Telecom, CNET, for providing the DFB lasers.

\section{REFERENCES}

[1] M. Jinno, "All optical signal regularizing/regeneration using a nonlinear fiber Sagnac interferometer switch with signal-clock walk-off," J. Lightwave Technol., vol. 12, pp. 1648-1659, Sept. 1994. 


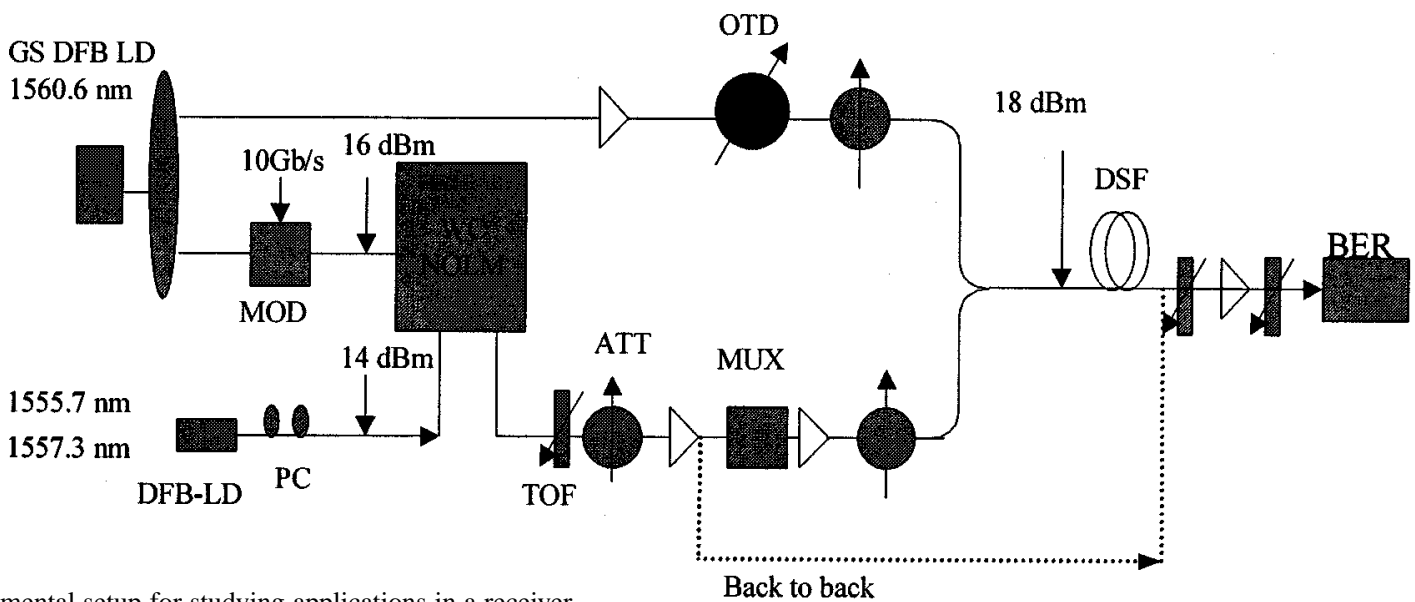

Fig. 15. Experimental setup for studying applications in a receiver.

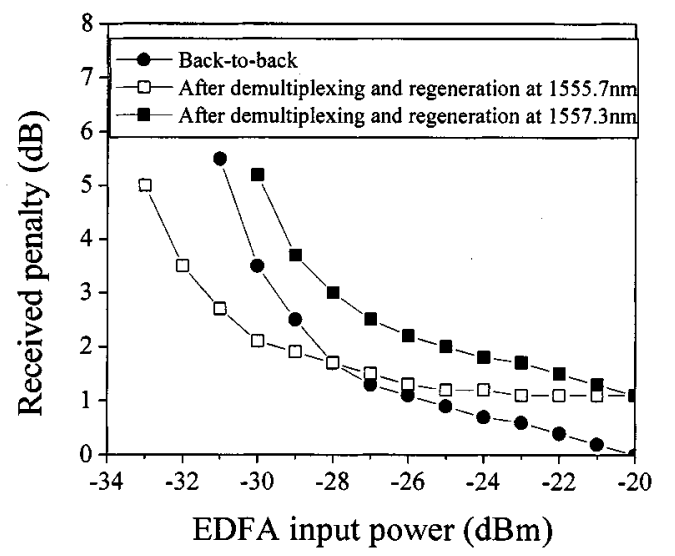

Fig. 16. Received penalty as a function of the EDFA input power.

[2] T. Yamamoto, E. Yoshida, and M. Nakazawa, "Ultrafast nonlinear optical loop mirror for demultiplexing $640 \mathrm{Gbit} / \mathrm{s}$ TDM signals," Electron. Lett., vol. 34, pp. 1013-1014, May 14, 1998.

[3] D. Phillips, A. D. Ellis, H. J. Thiele, R. J. Manning, and A. E. Kelly, “40 $\mathrm{Gb} / \mathrm{s}$ all-optical data regeneration and demultiplexing with long pattern lengths using a semiconductor nonlinear interferometer," Electron. Lett., vol. 34, pp. 2340-2342, Nov. 26, 1998.

[4] M. Vaa, B. Mikkelsen, K. S. Jepsen, and K. E. Stubkjaer, "Bit error rate assessment of $80 \mathrm{~Gb} / \mathrm{s}$ all-optical demultiplexing by a monolithically integrated Mach-Zehnder interferometer with semiconductor optical amplifiers," in ECOC'97, Edinburgh, Scotland, U.K., pp. WE1B-4-1438.

[5] T. Morioka, H. Takara, S. Kawanishi, T. Kitoh, and M. Saruwatari, "Error-free $500 \mathrm{~Gb} / \mathrm{s}$ all-optical demultiplexing using low-noise, low-jitter supercontinuum short pulses," Electron. Lett., vol. 32, pp. 833-834, Apr. 25, 1996.

[6] A. V. Mamyshev, "All-optical data regeneration based on self-phase modulation effect," in ECOC'98, Madrid, Spain, Sept. 20-24, 1998.

[7] D. M. Patrick and A. D. Ellis, " $10 \mathrm{GHz}$ pulse train derived from a $\mathrm{CW}$ DFB laser using crossphase modulation in an optical fiber," Electron. Lett., vol. 29, pp. 1391-1392, July 22, 1993.

[8] B. Olsson, P. Öhlen, L. Rau, and D. J. Blumenthal, "A simple and robust $40 \mathrm{~Gb} / \mathrm{s}$ wavelength conversion converter using fiber cross-phase modulation and optical filtering," IEEE Photon. Technol. Lett., vol. 12, pp. 846-848, July 2000.

[9] St. Fishcher, M. Dülk, E. Gamper, W. Vogt, W. Hunziker, E. Gini, H. Melchior, A. Buxens, H. N. Poulsen, and A. T. Clausen, "All-optical regenerative OTDM add-drop multiplexing at $40 \mathrm{~Gb} / \mathrm{s}$ using monolithic InP Mach-Zehnder interferometer," IEEE Photon. Technol. Lett., vol. 12, pp. 335-337, Mar. 2000.

[10] G. P. Agrawa, Nonlinear Fiber Optics, 2nd ed. San Diego, CA: Academic, 1995.

[11] J. Yu, X. Zheng, C. Peucheret, A. T. Clausen, H. N. Poulsen, and P. Jeppesen, "All-optical wavelength conversion of short pulses and NRZ signals based on a nonlinear optical loop mirror," J. Lightwave Technol., vol. 18, pp. 1007-1017, July 2000.

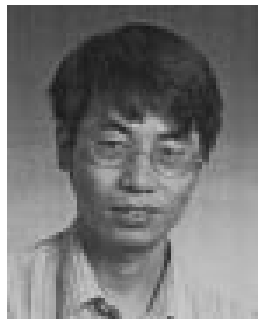

Jianjun Yu was born in Hunan, China, in 1968. He received the B.S. degree in optics from Xiangtan University, Hunan, in 1990 and the M.E. and Ph.D. degrees in optical communications from Beijing University of Posts \& Telecommunications, Beijing, China, in 1996 and 1999, respectively.

$\mathrm{He}$ joined Research Center COM, Technical University of Denmark, as a postdoctoral fellow in June 1999, where he researched high-speed optical communication systems and networks. In December 1999, he was named an Assistant Research Professor. In February 2001, he joined Agere Systems, Murray Hill, NJ. His research interests include the generation of WDM short pulse optical source, fiber nonlinear in high-speed WDM/OTDM optical communication systems, all-optical signal procession, wavelength conversion, and the application of semiconductor optical amplifier in optical networks.

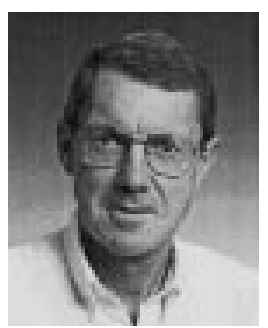

Palle Jeppesen (M'69) was born in Vordingborg, Denmark, on August 6, 1941. He received the M.Sc., Ph.D., and Dr.Sc. degrees in electrical engineering from Technical University of Denmark, Lyngby, in 1967,1970 , and 1978, respectively.

From 1968 to 1969, he was a Research Associate at Cornell University, Ithaca, NY, and from 1969 to 1970 , a Project Engineer at Cayuga Associates, Ithaca, NY; at both places he did research in the field of GaAs Gunn effect microwave oscillators. From 1970 to 1998, he was an Assistant, Associate, Research and full Professor at EMI, Technical University of Denmark, first in microwave electronics, and since 1974, in optical communications. At EMI, he was Head of Optogroup from 1974 to 1988 and Head of the Center for Broadband Telecommunications from 1988 to 1998. From 1982 to 1984, he also worked as part time Manager of R\&D at NKT Elektronik, now Draka Denmark Optical Cable, Lucent Technologies Denmark, and Tellabs Denmark. From 1995 to 1998, he coordinated the participation of Technical University of Denmark in the EU ACTS project METON (METropolitan Optical Network). Since 1999, he has been a Professor in optical communications at Research Center COM where he is heading the Systems Competence Area. His research interests include high-speed WDM optical communication systems, in particular, dispersion maps and WDM devices. 